DOI: $10.24850 /$ j-tyca-2018-05-04

Artículos

\title{
Modelación hidrológica de la cuenca del río Ilave a partir de datos de precipitación observada y de satélite, periodo 2011-2015, Puno, Perú
}

Ricardo Zubieta ${ }^{1,2}$

Wilber Laqui ${ }^{2,3}$

Waldo Lavado ${ }^{2,4}$

${ }^{1}$ Instituto Geofísico del Perú (IGP), Subdirección de Ciencias de la Atmósfera e Hidrósfera (SCAH), Lima, Perú, rzubieta@igp.gob.pe

${ }^{2}$ Universidad Nacional Agraria La Molina (UNALM), Programa de Doctorado en Recursos Hídricos, Lima, Perú, 20140761@lamolina.edu.pe

${ }^{3}$ Autoridad Nacional del Agua (ANA), Autoridad Administrativa del Agua Titicaca, Puno, Perú, wilfer.lv@gmail.com

${ }^{4}$ Servicio Nacional de Meteorología e Hidrología, (SENAMHI), Lima, Perú, waldo.lavado@gmail.com

Autor para correspondencia: Ricardo Zubieta, rzubieta@igp.gob.pe

\section{Resumen}

Los datos obtenidos mediante satélites acerca de la precipitación pueden ser útiles en regiones donde ésta es muy heterogénea, como, por ejemplo, en los Andes, pues por lo general son escasamente monitoreadas. El objetivo de este estudio es precisar las características de las principales variables hidrológicas, y comprender el potencial de datos de precipitación estimados a partir del satélite en modelación hidrológica. Este artículo evalúa datos de precipitación obtenidos a partir de pluviómetros y satélites (productos TMPA V7, TMPA RT del satélite TRMM), como entrada al modelo hidrológico agregado GR2M, para simulación de caudales mensuales, en el periodo 2011-2015, en la cuenca del río Ilave, en el Altiplano peruano. Los resultados mostraron 
un claro déficit hídrico de caudales debido a la disminución de las precipitaciones en el periodo de avenidas ( 50\%); además de un aumento de la evapotranspiración en el periodo de estiaje ( 24\%). Los resultados del análisis de datos de precipitación también indicaron que los TMPA V7 presentan mayor similitud que TMPA RT, con respecto a la precipitación observada en el periodo de avenidas. Del mismo modo, los resultados de modelación hidrológica con GR2M indicaron un mayor rendimiento a partir de la lluvia observada (pluviómetros) y TMPA V7, los cuales presentan una mayor similitud con caudales observados; a diferencia de los TMPA RT, es posible que los inconvenientes en el rendimiento se debieran a una inadecuada lluvia en el balance hídrico.

Palabras clave: precipitación, modelación hidrológica, Andes, TRMM, satélite.

Recibido: 23/05/2017

Aceptado: $11 / 04 / 2018$

\section{Introducción}

En las últimas décadas, numerosos datos globales y cuasiglobales de precipitación basada en satélites, observaciones terrestres o empleados de salidas de modelos de circulación general se han desarrollado en diferente resolución temporal (Huffman et al., 2007). La precipitación es un componente importante del ciclo del agua y una entrada clave para las aplicaciones de modelado hidrológico. La cuantificación fiable de la distribución espacio-temporal de la lluvia es fundamental para el análisis de los fenómenos hidrológicos extremos, como sequías o inundaciones, en tiempo real o casi real. No obstante, las redes de medición (meteorológicas e hidrológicas) en regiones del Altiplano pueden ser escasas o incluso inexistentes. Además, la existencia de los Andes contribuye a la alta variabilidad espacial de las precipitaciones (Espinoza et al., 2009), manifestándose en que grandes cantidades de lluvia pueden precipitarse en pocos días, lo que incrementa el riesgo de inestabilidad del suelo, deslizamientos o inundaciones (Coscarelli \& 
Caloiero, 2012; Zubieta \& Saavedra, 2013; Zubieta, Saavedra, Silva, \& Giraldez, 2017a). Los productos de precipitación basados en satélites han facilitado cada vez más la estimación de precipitaciones en gran parte del mundo y se han convertido en una alternativa complementaria para aplicaciones hidrometeorológicas y estudios sobre el clima en regiones no monitoreadas. Entre ellas se encuentran los datos del Tropical Rainfall Measuring Mission (TRMM), Análisis Multi-Satélite de Precipitación (TMPA, por sus siglas en inglés) de Datos (Huffman, Adler, Bolvin, \& Nelkin, 2010). TRMM fue una misión que comenzó en 1997, entre el National Space Development Agency del Japón (JAXA) y la National Aeronautics and Space Administration (NASA) de los Estados Unidos de América, la cual llegó a su fin el 8 de abril de 2015, como parte de su desactivación, al haber agotado sus reservas de energía (https://pmm.nasa.gov/trmm/mission-end). De forma reciente, la utilidad de estos datos en cuencas andino-amazónicas ha sido evaluada mediante la comparación de datos estimados y observados tanto de lluvia como caudal, dando a conocer que datos de precipitación a partir de satélites pueden ser considerados como entrada, para la modelación de caudales en cuencas (Zulkafli et al., 2014; Zubieta, Getirana, Espinoza, \& Lavado, 2015; Milewski, Elikadri, \& Durham, 2015; Zubieta, Getirana, Espinoza, Lavado-Casimiro, \& Aragon, 2017b). Zulkafli et al. (2014) documentaron que el rendimiento obtenido de la modelación hidrológica tiende a ser insatisfactorio sobre regiones andinoamazónicas del norte del Perú y el sur del Ecuador. No obstante, se obtienen mejores resultados sobre regiones andino-amazónicas del sur del Perú, los cuales están asociados con estimaciones adecuadas de lluvia a partir de satélite (Zubieta et al., 2015).

Para evaluar la utilidad de los datos de precipitación basados en satélites es necesario investigar heterogeneidades temporales de la lluvia y sus impactos en el ciclo hidrológico. La variabilidad de los caudales en cuencas andinas es consistente con la variabilidad de las precipitaciones a escala estacional e interanual. Esto se presenta sobre todo en los Andes peruanos, que se caracteriza por diferentes regímenes de lluvias, debido a su extensión latitudinal y la presencia de la cordillera (Espinoza et al., 2009). Por ejemplo, las precipitaciones en la cuenca del río Ramis en el Altiplano peruano presentan tendencias negativas de $0.70 \mathrm{~mm} / a n ̃ o$, mientras las temperaturas máximas presentan tendencias positivas significativas promedio de $0.04{ }^{\circ} \mathrm{C} / \mathrm{año}$; asimismo, las temperaturas mínimas a nivel de cuenca muestran un incremento de $0.0004{ }^{\circ} \mathrm{C} /$ año (Belizario, 2015). Por otro lado, en los últimos años, El Altiplano ha sufrido graves eventos hidrológicos 
extremos, como intensas sequías en 2008 y 2010 (ANA, 2010), e inundaciones en 2012 (RPP Noticias, 2012).

El objetivo principal de este estudio es evaluar la capacidad de datos TRMM, productos TMPA V7 y TMPA RT (real time) de precipitaciones basadas en satélites, para representar campos de precipitación de utilidad, para la modelación hidrológica de la cuenca del río Ilave (CRI). La CRI se ubica en los Andes, del sur del Perú, en la región norte del Altiplano (69.4 oeste-70.38 oeste; 17.14 sur-15.8 sur), con área de drenaje de $7790 \mathrm{~km}^{2}$; con altitudes que van de 3805 a $5400 \mathrm{~m}$ de altitud, y una altitud media de 4300 msnm (Figura 1a-b). La precipitación anual media a lo largo de la CRI es de entre 494 y 888 $\mathrm{mm} /$ año, además presenta un caudal promedio de $32 \mathrm{~m}^{3} / \mathrm{s}$. La amplitud del ciclo anual de precipitación es relativamente grande, con valores máximos entre diciembre y febrero, y mínimos entre junio y agosto.
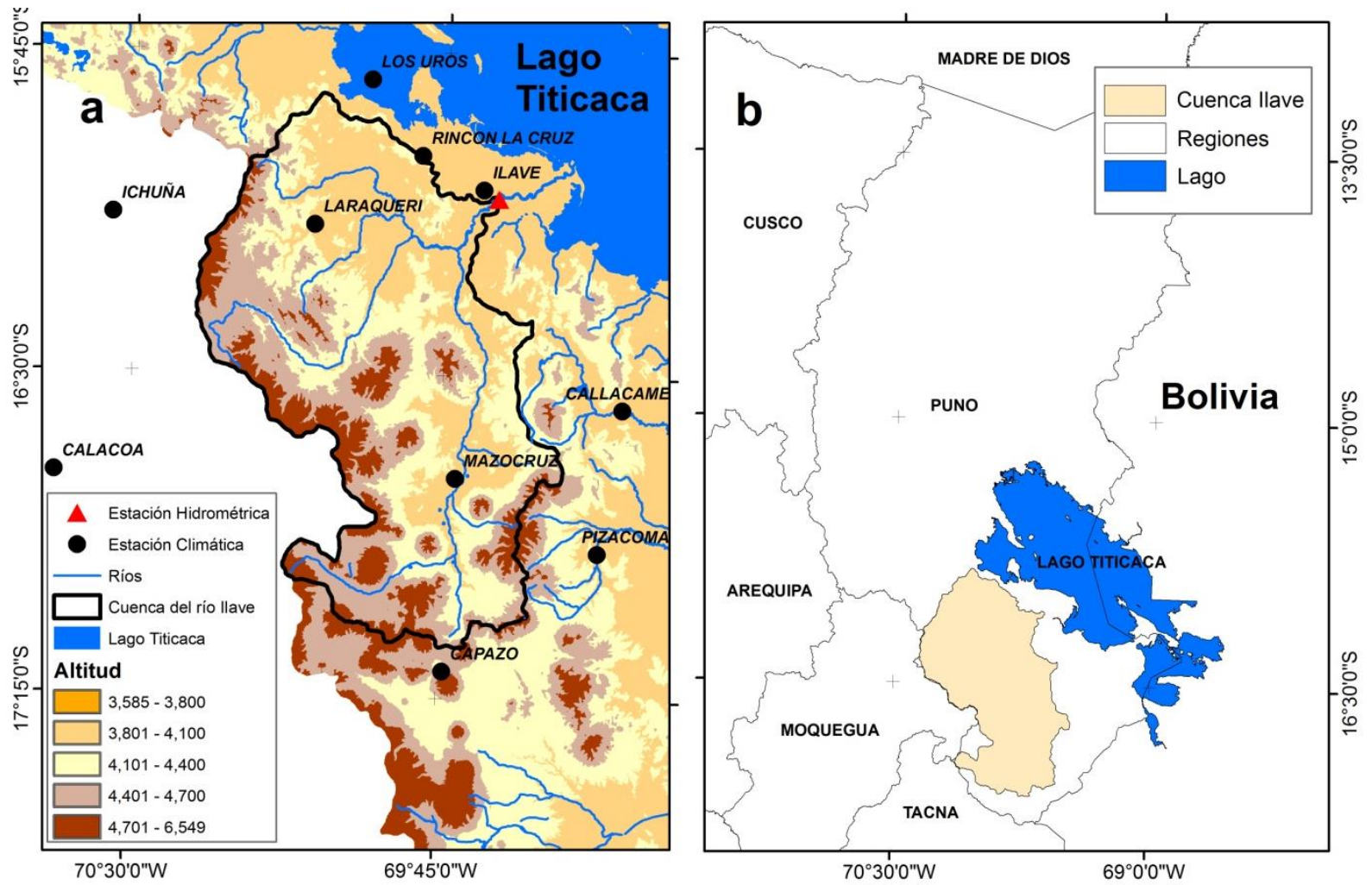

Figura 1. a) Ubicación de estaciones climáticas; b) ubicación de la cuenca del río Ilave.

También se consideraron los datos de precipitación observada, para efectos de comparación con las estimaciones satelitales. Cada conjunto 
de datos de precipitación (observado y satelital) se utilizan como entrada para el modelo GR2M (Niel, Paturel, \& Servat, 2003), el cual se basa en el modelo GR2M (Edijatno \& Michel, 1989; Kabouya, 1990), que fue revisado por Makhlouf y Michel (1994), para simulaciones de flujo fluvial y su comparación con caudales observados. GR2M se ha empleado para simular el comportamiento hidrológico de numerosas cuencas andino-amazónicas en el Perú (Lavado, Labat, Guyot, Ronchail, \& Ordoñez 2009; Lavado, Labat, Guyot, \& Ardoin-Bardin, 2011; Vera \& Felipe, 2011). Recientemente, Lujano, Felipe, Lujano y Quispe (2015) demostraron que los datos TMPA son factibles de emplear como entrada para el modelo GR2M en la cuenca del río Ramis, la cual se ubica al noroeste de la CRI.

\section{Datos}

Se recopilaron datos de precipitación diaria de 11 estaciones de lluvia del Servicio Nacional de Meteorología e Hidrología (SENAMHI). El periodo de datos seleccionado para el análisis hidrológico fue entre 2010 y 2015. No obstante, para garantizar la máxima disponibilidad de datos pluviométricos e hidrométrica se seleccionó un periodo común (20112015) (Figura 1b, Tabla 1). Para evaluar su calidad, esta base de datos, compuesta por un total de 10 estaciones de lluvia sobre una base mensual, se sometió al método de vector regional (RVM, por sus siglas en inglés), que utiliza el concepto de precipitación media extendida al periodo de estudio (Hiez, 1977; Brunet-Moret, 1979; Vauchel, 2006).

Tabla 1. Características generales de estaciones climáticas.

Latitud, longitud, altitud, lluvia, temperatura máxima ( $T$ máx), temperatura mínima ( $T$ mín) y porcentaje de la disponibilidad de datos en el periodo 2011-2015.

\begin{tabular}{|l|c|c|c|c|c|c|c|}
\hline Nombre & Latitud & Longitud & $\begin{array}{c}\text { Altitud } \\
\text { (msnm) }\end{array}$ & $\begin{array}{c}\text { Lluvia } \\
\mathbf{( m m )}\end{array}$ & $\begin{array}{c}\boldsymbol{T} \\
\text { máx }\end{array}$ & $\begin{array}{c}\boldsymbol{T} \\
\text { mín }\end{array}$ & $\begin{array}{c}\text { Disponibi- } \\
\text { lidad de } \\
\text { datos (\%) }\end{array}$ \\
\hline Capazo & -17.19 & -69.74 & 4530 & 464 & 13.6 & -6.3 & 99.8 \\
\hline Mazo Cruz & -16.74 & -69.72 & 4003 & 564 & 17.5 & -5.0 & 99.9 \\
\hline Laraqueri & -16.15 & -70.07 & 3900 & 788 & 16.7 & -1.6 & 99.8 \\
\hline Los Uros & -15.82 & -69.94 & 3808 & 810 & 15.8 & 3.9 & 99.9 \\
\hline
\end{tabular}


Tecnología y

Ciencias ฐึgua

\begin{tabular}{|l|c|c|c|c|c|c|c|}
\hline Rincón La Cruz & -15.99 & -69.81 & 3935 & 872 & 15.3 & 2.7 & 99.9 \\
\hline Ilave & -16.07 & -69.66 & 3871 & 647 & 15.3 & 1.5 & 99.9 \\
\hline Callacame & -16.57 & -69.32 & 3951 & 413 & 14.0 & 1.0 & 99.8 \\
\hline Pizacoma & -16.91 & -69.37 & 3930 & 583 & 17.0 & -0.9 & 99.9 \\
\hline Ichuña & -16.13 & -70.55 & 3800 & 595 & 20.1 & 2.5 & 99.9 \\
\hline Calacoa & -16.74 & -70.68 & 3260 & 386 & 25.0 & 4 & 99.8 \\
\hline
\end{tabular}

El método de mínimos cuadrados se aplicó para encontrar un índice pluviométrico anual regional $Z i$ y una precipitación media extendida. Este cálculo puede realizarse minimizando la suma de la ecuación (1), en donde $P_{i j}$ representa la lluvia anual para la estación $j, P_{j}$ es la precipitación media extendida; $i$, el índice años; $j$, el índice de la estación; $N$, el número de años, y $M$ es el número de estaciones. Por último, la serie de datos de $Z i$ se llama vector regional anual de los índices pluviométricos:

$\sum_{i=1}^{N} \sum_{\mathrm{j}=1}^{\mathrm{M}}\left(\frac{\mathrm{P}_{\mathrm{ij}}}{\mathrm{P}_{\mathrm{j}}}-\mathrm{Z}_{\mathrm{i}}\right)$

De este modo, se consideró una misma zona climática de la CRI, experimentando un mismo régimen de lluvias; se asumió que la precipitación anual en las estaciones de la cuenca mostró la proporcionalidad entre las estaciones; TRMM provee datos derivados entre sus productos, los cuales son evaluados en este estudio tanto:

1. TMPA 3B42 versión 7, que se obtiene a partir de un conjunto de datos de muchos sensores a bordo de satélites de precipitación tanto en tiempo real y casi-real (datos TMPA 3B42 están disponibles en https://pmm.nasa.gov/data-access/downloads/trmm).

TMPA muestra la experiencia más exitosa, ya que su procesamiento ha sido perfeccionado, al emplear datos de mejor escala en relación con otras fuentes de datos, siendo primordial crear promedios a nivel espacio-temporal que sean apropiados para la aplicación del usuario (Huffman et al., 2010).

2. Otro conjunto de datos son las estimaciones de precipitación TMPART. Estos datos en relación con TMPA V7 no incorporan mediciones de calibración de estaciones de lluvia, las cuales son incorporadas más de un mes después que de los datos de satélite. 
Tanto TMPA V7 y TMPA RT se caracterizan por proporcionar datos de precipitación con resolución espacial de $0.25^{\circ} * 0.25^{\circ}$ y resolución temporal de tres horas.

Se recopilaron datos de precipitación diaria de 11 estaciones de lluvia del Servicio Nacional de Meteorología e Hidrología (SENAMHI). El periodo de datos seleccionado para el análisis hidrológico fue entre los años 2010 y 2015. No obstante, para garantizar la máxima disponibilidad de datos pluviométricos e hidrométrica, se seleccionó un periodo común (2011-2015) (Figura 1b, Tabla 1).

Para evaluar su calidad, esta base de datos compuesta por un total de 10 estaciones de lluvia sobre una base mensual, la cual se sometió al método de vector regional (RVM), que utiliza el concepto de precipitación media extendida al periodo de estudio (Hiez, 1977; BrunetMoret, 1979; Vauchel, 2006). El método de mínimos cuadrados se aplicó para encontrar un índice pluviométrico anual regional $Z i$, y una precipitación media extendida. Para este fin, el cálculo puede realizarse minimizando la suma de la ecuación (1), en donde $P_{i j}$ es la precipitación anual en la estación j y $P_{j}$ es la precipitación media extendida; $i$, el índice años; $j$, el índice de la estación; $N$, el número de años, y $M$ es el número de estaciones. Por último, la serie de datos de $\mathrm{Zi}$ se llama vector regional anual de los índices pluviométricos.

\section{Metodología}

El GR2M es un modelo agregado que funciona a paso de tiempo mensual y requiere información promedio de variables climáticas para toda el área de drenaje hasta la estación Puente Carretera Ilave (Figura 1a). Ante el número reducido de estaciones (10) e incertidumbre en algunas regiones no monitoreadas de la cuenca, se procedió a interpolar datos de precipitación a la misma resolución de TMPA $\left(0.25^{\circ} * 0.25^{\circ}\right)$ mediante el método Kriging, aprovechando la interrelación que hay entre las estaciones ubicadas dentro y fuera de la cuenca. El método seleccionado en este estudio es el Kriging ordinario, que consiste en cuantificar el supuesto de que las mediciones cercanas tienden a ser más similares que otras más alejadas. La información detallada acerca 
del método de interpolación Kriging puede encontrarse en Lichtenstern (2013). Finalmente, se determinaron los valores promedio de precipitación para cada mes y para toda la cuenca durante el periodo 2010-2015.

Con base en la disponibilidad de datos de temperatura máxima y mínima, la variable evapotranspiración (ETP) se estimó con el modelo de Hargreaves y Samani (1985), cuyo cálculo se muestra en la ecuación (2).

$$
E T P=0.0023\left(T_{m}+17.8\right)\left(T_{\max }-T_{\min }\right)^{0.5} * R a
$$

donde ETP es evapotranspiración ( $\mathrm{mm} /$ día); $T_{m}$, temperatura media $\left({ }^{\circ} \mathrm{C}\right) ; T_{\text {máx }}$, temperatura máxima $\left({ }^{\circ} \mathrm{C}\right) ; T_{\text {mín }}$, temperatura mínima $\left({ }^{\circ} \mathrm{C}\right)$, y $R a$ es radiación extraterrestre $(\mathrm{mm} / \mathrm{día})$. Este método proporciona una apropiada aproximación con respecto a datos obtenidos por lisímetros en regiones del norte del Altiplano (mayor humedad), siendo éstos similares a aquellos obtenidos por la ecuación de FAO Penman-Monteith (García, Raes, Allen, \& Herbas, 2004). De hecho, estudios realizados en el Altiplano boliviano empleando variedades de pastizal (por encima de 3 $000 \mathrm{msnm}$ ) como cultivo de referencia sugieren que los métodos de Penman-Monteith y Hargreaves-Samani concuerdan de modo apropiado con datos a partir de lisímetros (García, Raes, \& Jacobsen, 2003; García et al., 2004; Vacher, Imana, \& Canqui, 1994). No obstante, Hargreaves y Samani (1985) tienden a ligeramente subestimar datos observados en la región sur del Altiplano, donde los factores aerodinámicos que afectan la evapotranspiración son más importantes que en el norte del Altiplano debido a la llanura y a un aumento de la aridez (García et al., 2004).

GR2M depende de dos parámetros: $X 1$ (máxima capacidad de almacenamiento del reservorio en $\mathrm{mm}$ ) y $X 2$ (coeficiente de intercambio de agua subterránea, adimensional). Conceptualmente, el modelo se basa en la transformación de la precipitación-escorrentía mediante dos funciones: de producción y de transferencia. El modelo GR2M es un modelo a dos reservorios, donde la función de producción se organiza alrededor de un reservorio llamado reservorio-suelo; la función de transferencia es regida por el segundo reservorio, llamado reservorio de agua gravitacional, donde el aporte es instantáneo al inicio del paso de tiempo, luego el reservorio se vacía de manera gradual. El nivel de este reservorio determina el caudal que puede liberar (Figura 2). 


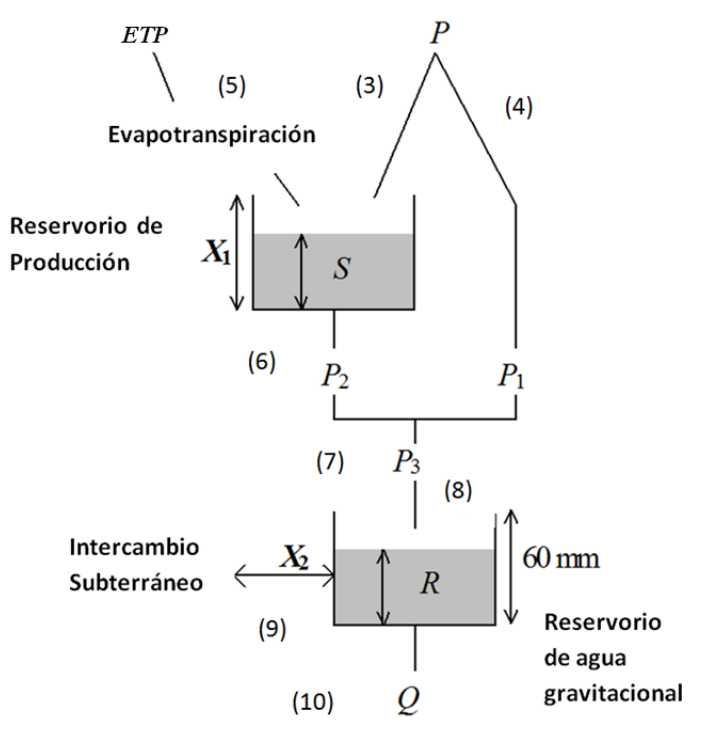

Figura 2. Modelo GR2M de Mouelhi (2003).

Fuente: Cemagref (www.cemagref.fr).

Las variables de entrada $(P)$ y salida (ETP) del reservorio-suelo (capacidad máxima $X 1$ ) se calculan con base en el almacenamiento del reservorio de producción $(S)$. Una parte de la precipitación $P 1$ hace referencia a la diferencia entre $P$ y la precipitación que ingresa en el suelo, y otra parte $P 2$ hace referencia a la cantidad de agua que contribuye al intercambio subterráneo. La suma tanto de $P 1$ y $P 2$ corresponde a la precipitación efectiva $P 3$, que ingresa en el reservorio de agua gravitacional $(R)$ (capacidad máxima fijada en $60 \mathrm{~mm}$ ). El intercambio subterráneo $(R 2)$ se incluye en el modelo $X 2$. Por último, como resultado para el caudal de salida, el reservorio de agua gravitacional se libera de acuerdo con una función cuadrática. La optimización del modelo responde a dos parámetros: $X 1$, capacidad del reservorio-suelo en milímetros; y $X 2$, coeficiente de intercambios subterráneos (adimensional). Para ello, se realizó una calibración manual previa, para emplear la herramienta SOLVER de Excel.

Para evaluar el rendimiento del modelo se utilizaron las siguientes funciones objetivo: coeficiente de Nash-Sutcliffe (NS); diferencia entre volúmenes calculados y observados mediante el error en el volumen $(\Delta V)$, y coeficiente de determinación mostrados $\left(\rho_{Q_{o b s}, Q_{c a l}}\right)$ en las ecuaciones (11), (12) y (13): 


$$
\begin{aligned}
& N S=1-\frac{\sum_{t=1}^{n t}\left(Q_{o b s}(t)-Q_{c a l}(\mathrm{t})\right)^{2}}{\sum_{t=1}^{n t}\left(Q_{o b s}(t)-\overline{Q_{o b s}}\right)^{2}} \\
& \Delta \mathrm{V}=\frac{\sum\left(Q_{o b s}(t)\right)-\sum\left(Q_{o b s}(t)\right)}{\sum\left(Q_{o b s}(t)\right)} \\
& \rho_{Q_{o b s}, Q_{c a l}}=\frac{\sigma_{Q_{o b s}, Q_{c a l}}}{\sigma_{Q_{o b s}} \cdot \sigma_{Q_{c a l}}}
\end{aligned}
$$

Donde $Q_{o b s}$ es el caudal observado; $Q_{c a l}$, el caudal estimado; $\sigma_{Q_{o b s}, Q_{c a l}}$, la covarianza entre $Q_{o b s}$ y $Q_{c a l}$; por último, $\sigma_{Q_{o b s}}$ y $\sigma_{Q_{c a l}}$ representan la desviación estándar de las variables $Q_{o b s}$ y $Q_{c a l}$, respectivamente.

\section{Resultados y discusión}

En términos promedio, el análisis visual del régimen de evapotranspiración estimada para el periodo 2011-2015 no muestra variaciones significativas durante el periodo de avenidas $(\sim 166 \mathrm{~mm}$, diciembre-febrero); no obstante, hay un incremento aproximado de 64 a $80 \mathrm{~mm}$ durante el periodo de estiaje. Basado en el balance hídrico, esto se asocia con la reducción de la precipitaciones identificada a lo largo del periodo de estudio ( 50\%) (Figura 3a). La precipitación media anual en la CRI indica que TMPA V7 (836.4 mm) y TMPA RT (1 $022.5 \mathrm{~mm})$, sobreestiman datos observados $(688.0 \mathrm{~mm})$ en 21.6 y $48.6 \%$, respectivamente. 

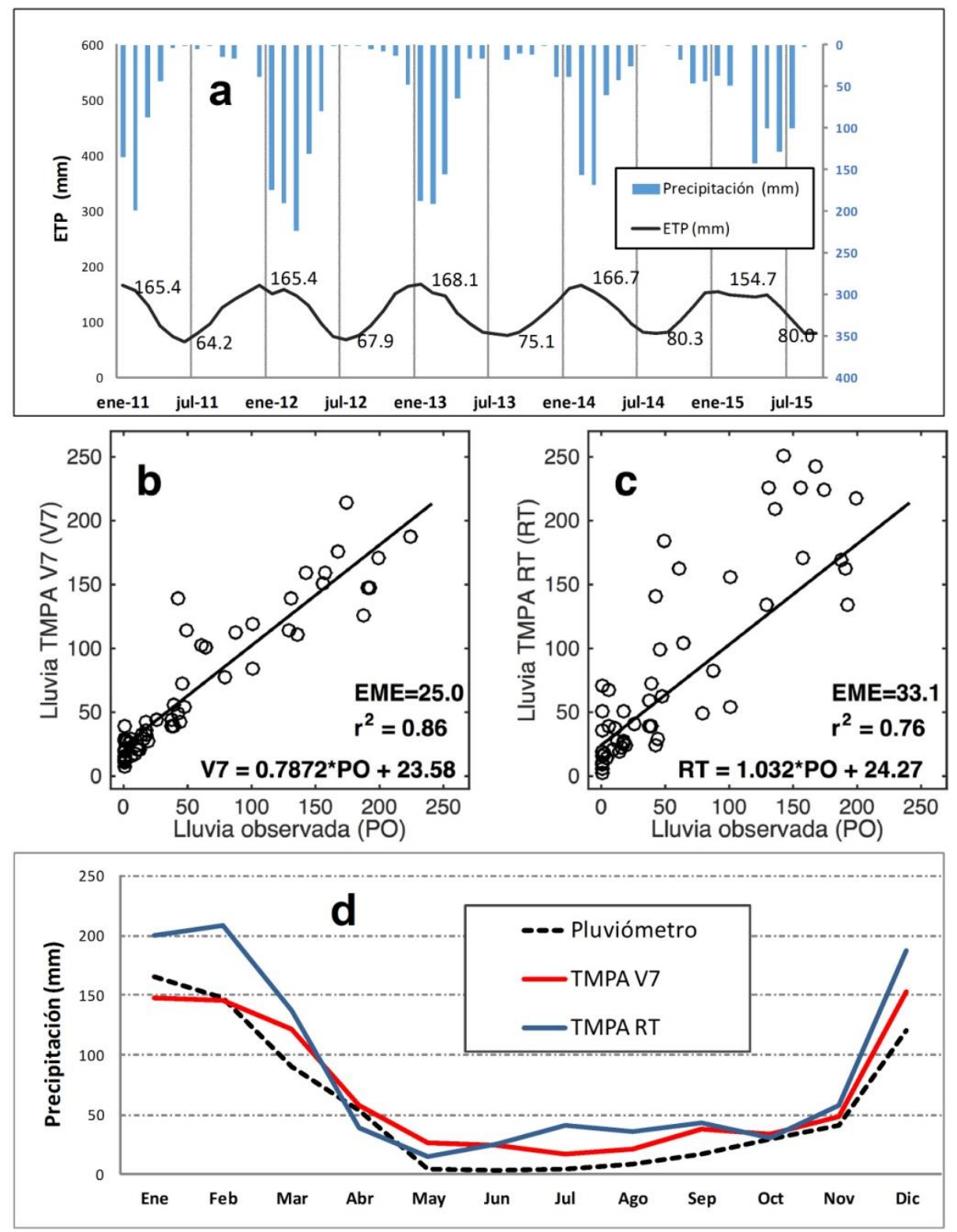

Figura 3. a) Comparación entre evapotranspiración y lluvia observada, diagramas de dispersión de los productos de lluvia; b) TMPA V7, y c) TMPA RT en relación con la Iluvia observada; d) Iluvia promedio mensual para cada producto de lluvia.

El análisis de la precipitación mensual de la CRI muestra que las estimaciones de datos TMPA V7 son más realistas en relación con los datos observados al ser identificado un alto coeficiente de determinación entre ellos $\left(r^{2}=0.86\right)$ y menor error estándar (EME $\left.=25 \mathrm{~mm}\right)$ (Figura 3b). Sin embargo, TMPA V7 muestra diferencias con respecto a datos observados en las estimaciones de lluvia por encima de $50 \mathrm{~mm} / \mathrm{mes}$. 
Asimismo, datos TMPA RT muestra mayores diferencias que TMPA V7 para estimaciones por encima de $50 \mathrm{~mm} / \mathrm{mes}$, a pesar de presentar un coeficiente de determinación relativamente aceptable $\left(r^{2}=0.76\right)$ y un mayor error (EME = $33.1 \mathrm{~mm}$ ) (Figura $3 \mathrm{c}$ ).

Para un análisis temporal de la lluvia se calculó la lluvia promedio mensual para cada conjunto de datos (pluviómetros, TMPA V7, TMPA $\mathrm{RT}$ ). El análisis corroboró que las estimaciones TMPA V7 son las más aproximadas en relación con los datos observados durante el periodo de avenidas (diciembre-marzo) (Figura 3d). Pero TMPA V7 muestra tendencia a sobreestimar la lluvia observada durante el periodo de estiaje en $\sim 22 \mathrm{~mm}$ (mayo-septiembre). Por otro lado, datos TMPA RT muestran tendencia a sobreestimar datos observados predominantemente a lo largo de todo el año.

Las diferencias identificadas en las estimaciones satelitales con respecto a datos de lluvia observada pueden estar asociadas con variaciones observacionales y/o provenientes de datos de sensores en la entrada de datos al algoritmo utilizado para la estimación de precipitación satelital (Huffman et al., 2007). De hecho, se encontraron resultados similares en la cuenca del río Ramis, en el Altiplano peruano (Lujano et al., 2015), u otras regiones de los Andes del Perú y Ecuador (Zulkafli et al., 2014; Zubieta et al., 2015).

Para el proceso de calibración del modelo se realizó una evaluación previa en la selección del número de años de los periodos de calibración y validación. En condiciones promedio, para un periodo de calibración de un año (periodo de validación cuatro años), el rendimiento del modelo tiende a ser mayor que al seleccionar 2 o 3 años (periodo de validación 3 y 2 años), los cuales tienen reducciones de 30 y $45 \%$ en el rendimiento, respectivamente. Por tal motivo, para la calibración del modelo, tanto de lluvia observada y de satélite, se consideró sólo el periodo 2011, mientras que para la validación se consideró el periodo de 2012-2015.

Las variables de entrada al modelo fueron los valores promedio mensuales de precipitación, evapotranspiración potencial y caudales. El proceso de optimización se basó en la obtención de parámetros idóneos ( $X 1$ y $X 2)$, del modelo de acuerdo con la ecuación (10), empleando como variable de entrada al modelo, tanto datos de lluvia observada como datos satelitales (TMPA V7, TMPA RT). Los valores $X 1$ y $X 2$ optimizados para cada conjunto de datos de precipitación se muestran en la Tabla 2, donde también se observan los valores encontrados para el coeficiente de Nash, desbalance hídrico y coeficientes de correlación, 
al realizar la evaluación de datos de caudales modelados con respecto a caudales observados.

Tabla 2. Parámetros de calibración, coeficientes de rendimiento Nash-Sutcliffe, diferencias entre volúmenes, y coeficiente de determinación entre caudales calculados y observados.

\begin{tabular}{|l|c|c|c|}
\hline \multicolumn{1}{|c|}{ Parámetro } & $\begin{array}{c}\text { Lluvia } \\
\text { observada }\end{array}$ & TMPA V7 & TMPA RT \\
\hline X1: reservorio de producción $(\mathrm{mm})$ & 5.76 & 5.57 & 6.35 \\
\hline X2: parámetro de intercambio (mm) & 1.00 & 0.96 & 0.74 \\
\hline Coeficiente de Nash-Sutcliffe (NS) & 0.95 & 0.74 & 0.52 \\
\hline $\begin{array}{l}\text { \% de diferencia entre volúmenes } \\
\text { calculados y observados }(\Delta V)\end{array}$ & 5 & 23 & 41 \\
\hline Coeficiente de determinación $\left(R^{2}\right)$ & 0.85 & 0.64 & 0.45 \\
\hline
\end{tabular}

Similar al régimen de precipitaciones, el régimen de caudales observados muestra también un claro retroceso de 2011 a 2015 tanto en el periodo de avenidas o estiaje, y del patrón similar para cada serie de caudales estimadas a partir de datos de satélite (TMPA V7 y TMPA $\mathrm{RT}$ ). De acuerdo con el balance hídrico y el alto rendimiento del modelo GR2M en la estimación de caudales empleando datos observados, este déficit se debe a la disminución predominante de las precipitaciones, el cual también es identificado en la serie de caudales obtenidos a partir de satélite. Del mismo modo, está influenciado por el incremento de la evapotranspiración estimada en estiaje durante el periodo 2011-2015 (Figuras $4 a$ y $3 a$ ). 


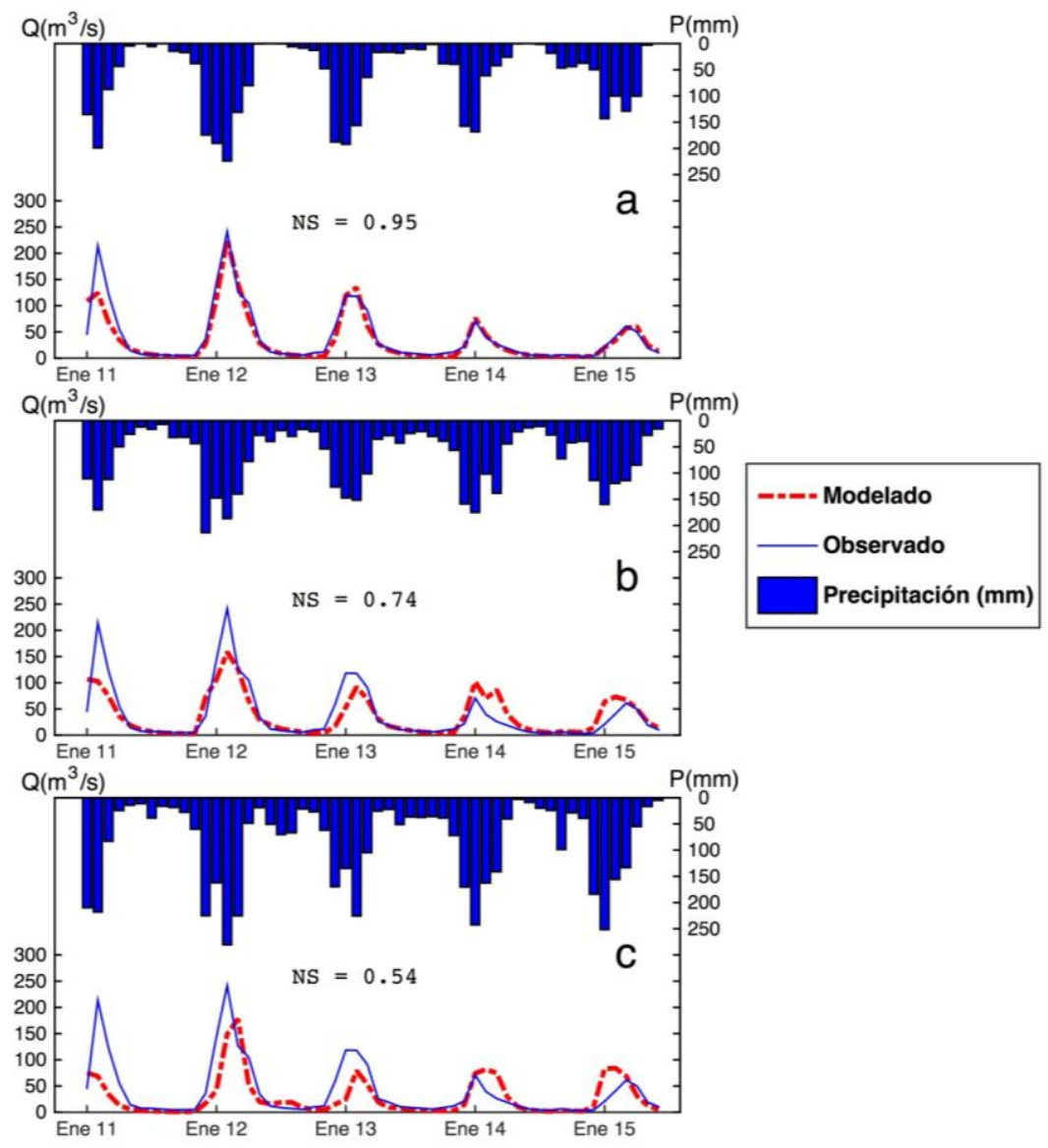

Figura 4. Caudales observados y modelados en la estación Puente Carretera Ilave desde enero de 2011 hasta junio de 2015. Los caudales modelados son calculados empleando datos de precipitación obtenidos a partir de a) pluviómetros; b) TMPA V7, y c) TMPA RT en la cuenca del río Ilave.

La disminución de las precipitaciones puede estar asociada con el incremento sustancial en la ocurrencia de frentes cálidos en el Altiplano, los cuales pueden aumentar la evaporación atmosférica, además de disminuir la vegetación (Thibeault, Seth, \& Garcia, 2010).

El análisis visual de los hidrogramas revela que los caudales obtenidos a partir de lluvia observada (pluviómetros) muestran mayor similitud con caudales observados registrados en la estación de aforo Puente Carretera Ilave ( $N S=0.95$ y $\Delta V=-11.5 \%)$, que se muestran en la Figura $4 a$. El régimen de precipitaciones es consistente en relación con los caudales obtenidos en el balance hídrico (ver Figura 4a). Las fluctuaciones estacionales en el régimen de caudales están muy bien representadas por el modelo GR2M, al emplear datos observados, 
principalmente durante el periodo de validación, tal es el caso de los caudales registrados en el periodo de avenidas 2012, 2014 y 2015.

No obstante, el rendimiento del modelo GR2M al utilizar datos TMPA V7 en la CRI es mínimamente aceptable, $N S=0.74, \Delta V=-7.3 \%$ a pesar de presentar un NS relativamente alto (Figura $4 \mathrm{~b}$ ), pues no tiene la capacidad de representar de manera adecuada todos los caudales picos, lo que podría deberse a la sobreestimación de la lluvia durante la temporada de inicio de máximas avenidas (diciembre-enero).

Sin embargo, puede reproducir con mejor aproximación caudales estacionales, sobre todo durante los periodos de estiaje. Se puede observar menor rendimiento al obtenido con TMPA V7 cuando se utiliza TMPA RT (NS $=0.52, \Delta V=-28 \%$ ) (Figura 4c). Basado en los resultados adecuados en la modelación, a partir de datos de lluvia observada, el muy bajo rendimiento al emplear datos TMPA RT se debe a una lluvia estimada por satélite inadecuada, caracterizada en valores promedio por una marcada sobrestimación con respecto a datos observados (pluviómetro) (31\%) y datos TMPA V7 (35\%) durante el periodo de avenidas (Figura 3d). Asimismo, esta sobrestimación también se aprecia durante el estiaje, la cual asciende hasta en $81 \%$ con respecto a la lluvia observada (pluviómetros) y $16 \%$ con respecto a la lluvia TMPA V7.

Los caudales mensuales 2011-2015 obtenidos a partir del satélite se compararon con datos observados provenientes de la estación Puente Carretera Ilave mediante diagramas de dispersión (Figura 5a-c), y muestran que las series generadas por el modelo que usa datos observados (pluviómetros) y TMPA V7 representan mejor los caudales observados, al presentar coeficientes de determinación aceptables $(0.85$ y 0.64 , respectivamente). Sin embargo, los caudales $\left(Q>75 \mathrm{~m}^{3} / \mathrm{s}\right)$ presentes de manera predominante durante el periodo de máximas avenidas (enero-marzo) presentan una mayor dispersión de los datos, a diferencia de otros periodos (estiaje durante Junio-Julio o inicio de precipitación entre Setiembre-diciembre). En general, esto sugiere una mejor aproximación de datos TMPA V7, siendo éstos los de mayor similitud a caudales observados. No obstante, los caudales simulados usando TMPA RT muestran un bajo coeficiente de determinación $\left(r^{2}=\right.$ $0.45)$. 

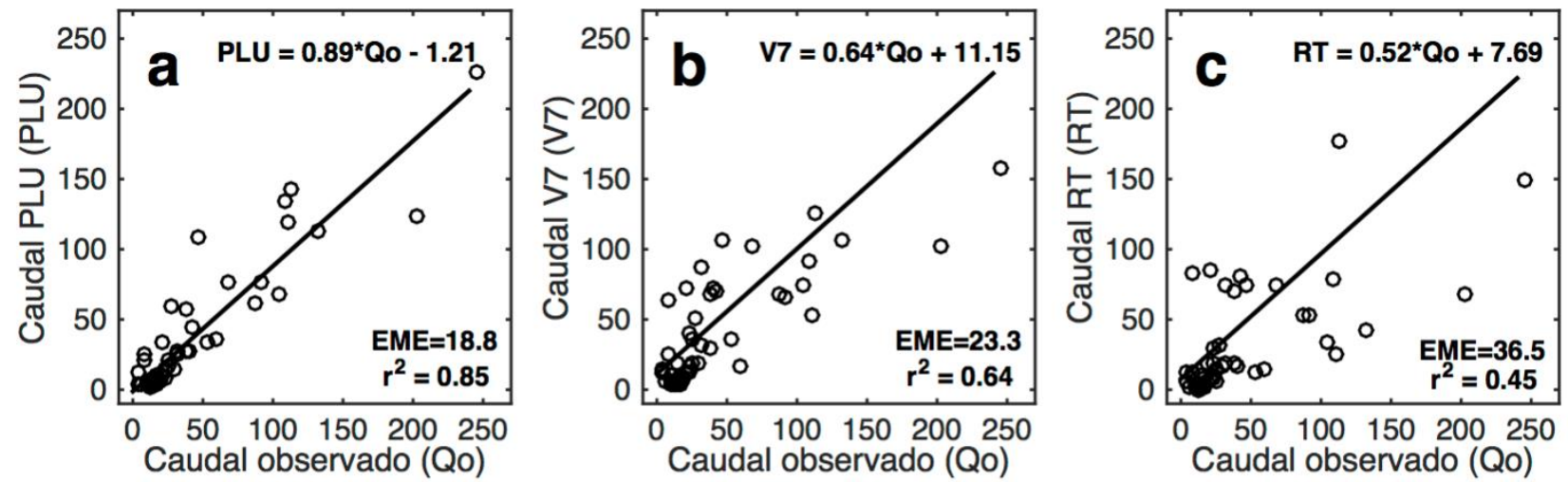

Figura 5. Caudales observados y modelados empleando a) Lluvia observada y lluvia (PLU) obtenida por satélites b) TMPA V7, c) TMPA RT.

La mayor similitud entre datos TMPA V7 y datos observados de lluvia, además de la sobrestimación de TMPA RT con respecto a datos observados (Figura $4 b-c$ ), son congruentes con el impacto reflejado en el parámetro de máxima capacidad de almacenamiento del reservorio $X 1$, pues estos parámetros obtenidos en el proceso de optimización muestran ser más similares cuando son provistos por TMPA V7 y datos observados (Tabla 2).

Por otro lado, los caudales modelados con GR2M empleando lluvia satelital (TMPA V7, TMPA RT) tienden a subestimar caudales observados. Esto se asocia principalmente con el proceso de optimización del modelo, pues un menor intercambio subterráneo (X2) es efectivo cuando se modela con lluvia satelital provista por TMPA V7 $(X 2=0.96)$ y TMPA RT $(X 2=0.74)$ (ver Tabla 2$)$. Esto sugiere un impacto negativo en la producción final de caudales a medida que $X 2$ sea menor, lo cual implica que aunque la precipitación satelital tienda a sobrestimar lluvia observada en el periodo de avenidas, los resultados del modelo empleando esta lluvia tienden a subestimar los caudales observados.

\section{Conclusiones}


Se ha identificado un claro déficit en el régimen hídrico de caudales del río Ilave en el Altiplano peruano para el periodo 2011-2015. De acuerdo con el balance hidrológico realizado, el déficit hídrico se debe a la disminución de las precipitaciones en periodo de avenidas ( 50\%) además de un aumento de la evapotranspiración en el periodo de estiaje ( 24\%).

Dos conjuntos de datos de lluvia mensual basados en el satélite TRMM (TMPA V7, TMPA RT) se compararon con datos de precipitación observada, basada en pluviómetros sobre la cuenca del río Ilave. En condiciones promedio, y a partir de análisis estadístico, TMPAV7 presenta una mayor asociación con respecto a la precipitación observada, principalmente durante el periodo de avenidas (eneromarzo); no obstante, TMPA V7 muestra tendencia a sobreestimar lluvia observada durante el periodo de estiaje (mayo-setiembre). Por otro lado, datos basados en TMPA RT muestran de manera predominante la sobreestimación a lo largo del año.

Para investigar la ventaja de datos satelitales en hidrología, los conjuntos de datos de precipitación observada basada en pluviómetros, TMPA V7 y TMPA RT se emplearon como variable de entrada al modelo hidrológico GR2M. El análisis de los hidrogramas revela que los caudales obtenidos a partir de lluvia observada (pluviómetros) muestra mayor similitud con caudales observados en la estación de aforo Puente Carretera Ilave (NS $=0.95$ ). Cabe resaltar que el rendimiento del modelo GR2M al emplear datos TMPA V7 puede representar de forma adecuada el ciclo estacional de caudales (NS $=0.74)$, pero no presenta habilidad para representar de modo correcto caudales pico. Esto sugiere una inadecuada estimación de lluvia durante el mes de febrero, caracterizada por una subestimación de lluvia.

Se encontró un menor rendimiento al obtenido con TMPA V7 al usar TMPA RT ( $N S=0.54)$. Con base en resultados adecuados en la modelación, a partir de datos de lluvia observada, el muy bajo rendimiento al emplear datos TMPA RT se debe a una inadecuada lluvia estimada por satélite, caracterizada por una marcada sobrestimación con respecto a datos observados. De hecho, los caudales muestran que las series generadas por el modelo a partir de datos observados (pluviómetros) y TMPA V7 representan mejor los caudales observados durante el periodo de avenidas.

Errores en las simulaciones de caudal de GR2M pueden estar asociados principalmente con la entrada de datos de lluvia o evapotranspiración, además de la representación limitada de procesos físicos del modelo. Sin 
embargo, los resultados muestran que es posible utilizar datos obtenidos a partir de satélites y usarlos en modelación hidrológica de pequeñas cuencas en los Andes altiplánicos del Perú, para simular de modo apropiado los caudales.

Evaluar los datos de la nueva generación de estimaciones de lluvia proporcionada por el satélite GPM (Global Precipitation Measurement) (Schwaller \& Morris, 2011) es un área de estudio interesante para la hidrología. Este tipo de datos provee la posibilidad de tomar ventaja de datos subdiarios o subhorarios de precipitación para estimación de caudales de la misma resolución temporal usando modelos hidrológicos.

\section{Agradecimientos}

Los autores agradecen al Servicio Nacional de Meteorología e Hidrología (SENAMHI), que facilitó la información hidrometeorológica para la realización del presente trabajo de investigación. Asimismo, reconocen al GSFC/DAAC, NASA, por proveer los datos TMPA V7 y TMPA RT (https://pmm.nasa.gov/data-access/downloads/trmm). El primer autor también agradece al Msc. Juan Sulca por sus aportes en la revisión del artículo.

\section{Referencias}

ANA, Autoridad Nacional del Agua. (2010). Las condiciones de sequía y estrategias de gestión en el Perú. Informe Nacional del Perú. Lima, Perú: Autoridad Nacional del Agua.

Belizario, G. (2015). Efectos del cambio climático en la agricultura de la cuenca Ramis, Puno-Perú. Revista de Investigaciones Altoandinas, 17(1), 47-52. http://dx.doi.org/10.18271/ria.2015.77

Brunet-Moret, Y. (1979). Homogénéisation des précipitations. Cahiers ORSTOM, Série Hydrologie, 16(3), 3-4.

Coscarelli, R., \& Caloiero, T. (2012). Analysis of daily and monthly rainfall concentration in Southern Italy (Calabria region). Journal of Hydrology, 416, 145-156. DOI: 10.1016/j.jhydrol.2011.11.047

Edijatno, E., \& Michel, C. (1989). Un modèle pluie-débit journalier à trois paramètres. La Houille Blanche, 2, 113-121 
Espinoza, J. C., Ronchail J., Guyot, J. L., Cocheneau, G., Filizola, N., Lavado, W., De Oliveira, E., Pombosa, R., \& Vauchel, P. (2009). Spatiotemporal rainfall variability in the Amazon Basin Countries (Brazil, Peru, Bolivia, Colombia and Ecuador). International Journal of Climatology, 29(11), 1574-1594. Recuperado de https://doi.org/10.1002/joc.1791

García, M., Raes, D., \& Jacobsen, S. E. (2003). Evapotranspiration analysis and irrigation requirements of quinoa (Chenopodium quinoa) in the Bolivian highlands. Agricultural Water Management, 60(2), 119-134. Recuperado de https://doi.org/10.1016/S0378-3774(02)00162-2

García, M., Raes, D., Allen, R. G., \& Herbas, C. (2004). Dynamics of reference evapotranspiration in the Bolivian highlands (Altiplano). Agricultural and Forest Meteorology, 125(1-2), 67-82. Recuperado de https://doi.org/10.1016/j.agrformet.2004.03.005

Hargreaves, G. H., \& Samani, Z. A. (1985). Reference crop evapotranspiration from temperature. Transactions of the ASAE, 2, 9699.

Hiez, G. (1977). L'homogénéité des données pluviométriques. Cahier ORSTOM, Série Hydrologie, 14(2), 129-172.

Huffman, G., Adler, R., Bolvin, D., Gu, G., Nelkin, E., Bowman, K., Hong, Y., Stocker, E., \& Wolff, D. (2007). The TRMM multisatellite precipitation analysis (TCMA): Quasiglobal, multiyear, combined-sensor precipitation estimates at fine scales. Journal of Hydrometeorology, 8, 38-55. Recuperado de https://doi.org/10.1175/JHM560.1

Huffman, G. J., Adler, R. F., Bolvin, D. T., \& Nelkin, E. J. (2010). The TRMM multi-satellite precipitation analysis (TMPA). Satellite Rainfall. Applications for Surface Hydrology. DOI: 10.100/978-90-481-2915-7_1

Kabouya, M. (1990). Modélisation pluie-débit au pas de temps mensuel et annuel en Algérie septentrionale (tesis de doctorado). Université Toulouse, Paris, France.

Lavado, W., Labat, D., Guyot, J., Ronchail, J., \& Ordoñez, J. L. (2009). TRMM rainfall data estimation over the Peruvian Amazon-Andes basin and its assimilation into a monthly water balance model. International Association of Hydrological Sciences Publication, 333, 245-252.

Lavado, W., Labat, D., Guyot, J., \& Ardoin-Bardin, S. (2011). Assessment of climate change impacts on the hydrology of the Peruvian AmazonAndes basin. Hydrological Processes, 25(24), 3721-34. Recuperado de https://doi.org/10.1002/hyp.8097

Lichtenstern, A. (2013). Kriging methods in spatial statistics (tesis de licenciatura). Tecnische Universität München, München, Germany.

Lujano, E., Felipe, O., Lujano, A., \& Quispe, J. (2015). Validación de la precipitación estimada por satélite TRMM y su aplicación en la 
modelación hidrológica del río Ramis, Puno, Perú. Revista de investigaciones Altoandinas, 17(2), 221-228. Recuperado de http://dx.doi.org/10.18271/ria.2015.116

Makhlouf, Z., \& Michel, C. (1994). A two-parameter monthly water balance model for French watersheds. Journal of Hydrology, 162(3-4), 299-318. Recuperado de https://doi.org/10.1016/0022-1694(94)90233-X

Milewski, A., Elikadri, R., \& Durham, M. (2015). Assessment and Comparison of TMPA Satellite Precipitation Products in Variying Climate and Topographic Regimes in Morocco. Remote Sensing, 7(5), 56975717. DOI: $10.3390 /$ rs70505697

Niel, H., Paturel, J. E., \& Servat, E. (2003). Study of parameter stability of a lumped hydrologic model in a context of climatic variability. Journal of Hydrology, 278(1-4), 213-230. Recuperado de https://doi.org/10.1016/S0022-1694(03)00158-6

RPP Noticias. (2012). Puno: inundaciones afectaron más de 37 mil hectáreas de cultivos. Radio Programas del Perú. Recuperado de http://rpp.pe/peru/actualidad/puno-inundaciones-afectaron-mas-de-37mil-hectareas-de-cultivos-noticia-454127

Schwaller, M. R., \& Morris, K. R. (2011). A ground validation network for the global precipitation measurement mission. Journal of Atmospheric and Oceanic Technology, 28, 301-319. Recuperado de https://doi.org/10.1175/2010JTECHA1403.1

Thibeault, J. M., Seth, A., \& Garcia, M. (2010). Changing climate in the Bolivian Altiplano: CMIP3 projections for temperature and precipitation extremes. Journal of Geophysical Research, 115, D08103. DOI: 10.1029/2009JD012718

Vacher, J., Imana, E., \& Canqui, E. (1994). Las características radiativas y la evapotranspiración potencial en el Altiplano boliviano. Convenio sistemas de cultivo en la región de Cochabamba. Revista de Agricultura, 50(24), 4-11.

Vauchel, P. (2006). Hydraccess, software hidrológico. Recuperado de http://www.so-hybam.org/index.php/esl/Software/Hydraccess

Vera, H., \& Felipe, O. (2011). Modelización de los caudales medios mensuales en la cuenca del río Ramis. Revista Peruana Geo-Atmosférica RPGA, (3), 116-124.

Zubieta, R., \& Saavedra, M. (2013). Distribución espacial del índice de concentración de precipitación diaria en los Andes centrales peruanos: valle del río Mantaro. Revista del Encuentro Científico Internacional ECIPeru, 9(2), 61-70.

Zubieta, R., Getirana, A., Espinoza, J. C., \& Lavado W. (2015). Impacts of satellite-based precipitation datasets on rainfall-runoff modeling of the 
western Amazon basin of Peru and Ecuador. Journal of Hydrology. DOI: 10.1016/j.jhydrol.2015.06.064

Zubieta, R., Saavedra, M., Silva, Y., \& Giraldez, L. (2017a). Spatial analysis and temporal trends of daily precipitation concentration in the Mantaro River basin. Central Andes of Peru. Stochastic Environmental Research and Risk Assessment. DOI: 10.1007/s00477-016-1235-5

Zubieta, R., Getirana, A., Espinoza, J. C., Lavado-Casimiro, W., \& Aragon, L. (2017b). Hydrological modeling of the Peruvian-Ecuadorian Amazon basin using GPM-IMERG satellite-based precipitation dataset. Hydrology and Earth System Sciences, 21(7), 3543-3555. Recuperado de https://doi.org/10.5194/hess-21-3543-2017

Zulkafli, Z., Buytaert, W., Onof, C., Manf, B., Tarnavsky, E., Lavado, W., \& Guyot, J. L. (2014). A comparative performance analysis of TRMM $3 B 42$ (TMPA) versions 6 and 7 for hydrological applications over AndeanAmazon River Basins. Journal of Hydrometeorology, 15, 581-592. DOI: 10.1175/JHM-D-13-094 